\title{
Design and Synthesis of New Benzofuran-1,2,3-Triazole Hybrid Preservatives and the Evaluation of Their Antifungal Potential Against White and Brown-Rot Fungi
}

\author{
Fahimeh Abedinifar, ${ }^{a}$ S. Morteza F. Farnia, ${ }^{\mathrm{a}}{ }^{*}$ Seyyed Khalil Hosseinihashemi, ${ }^{\mathrm{b}, *}$ \\ Abbas Jalaligoldeh, ${ }^{\mathrm{c}}$ Shahrbanoo Arabahmadi, ${ }^{\mathrm{d}}$ and Mohammad Mahdavi ${ }^{\mathrm{e}}$
}

\begin{abstract}
A series of novel benzofuran-1,2,3-triazole hybrids were synthesized and investigated as fungicidal preservatives. The compounds were evaluated for their antifungal potential against white-rot (Trametes versicolor), dry brown-rot (Poria placenta), and wet brown-rot (Coniophora puteana and Gloeophyllum trabeum) fungi, at different concentrations (500 ppm and $1000 \mathrm{ppm})$. The tests of the final products $(\mathbf{8 a}, \mathbf{8 b}, \mathbf{8 c}, \mathbf{8 d}, \mathbf{8 e}, \mathbf{8 f}$, and $8 \mathrm{~g})$ demonstrated that compound $\mathrm{N}-((1-(4-f)$ uorobenzyl)-1H-1,2,3-triazol4-yl)methyl)benzofuran-2-carboxamide (8f) at a concentration of 500 ppm was the most active against wet brown-rot C. puteana $(23.86 \%$ inhibition) and $G$. trabeum (47.16\% inhibition) fungi. However, testing demonstrated that compounds $\mathbf{8 a}, \mathbf{8 b}, \mathbf{8 c}, \mathbf{8 d}$, and $\mathbf{8 g}$ at a concentration of $500 \mathrm{ppm}$ did not exhibit acceptable antifungal effects against white-rot $T$. versicolor and dry brown-rot $P$. placenta fungi.
\end{abstract}

Keywords: Triazoles; Synthesis; Antifungal activity; White and brown-rot fungi

Contact information: a: Department of Chemistry, College of Science, University of Tehran, Tehran, Iran; b: Department of Wood Science and Paper Technology, Karaj Branch, Islamic Azad University, Karaj, Iran; c: Department of Horticulture, Karaj Branch, Islamic Azad University, Karaj, Iran; d: Department of Chemistry, University of Mazandaran, P. O. Box: 47416-95447, Babolsar, Iran; e: Endocrinology and Metabolism Research Center, Endocrinology and Metabolism Clinical Science Institute, Tehran University of Medical Sciences, Tehran, Iran; *Corresponding authors: mfarnia@ gmail.com and hashemi@kiau.ac.ir

\section{INTRODUCTION}

Carbamates, creosotes, isothiazolones, quaternary ammonium compounds, sulfamides, and triazoles are the main classes of metal-free organic fungicides used for wood protection (Reinprecht 2010). Many of these preservatives are effective against a specific class of microorganism; however, synergistic effects have been reported for a group of triazole fungicides, which help overcome this selectivity (Bauschhaus and Valcke 1995; Christen et al. 2014). Furthermore, triazoles have an added advantage of exhibiting high antifungal activity in both organic solvent- and water-based formulations (Bruns et al. 2005).

Triazole fungicides have great importance in agriculture and medicine and are commonly used in two categories: (1) triazole antifungal drugs, e.g., fluconazole, voriconazole, isavuconazole, itraconazole, etc., and (2) triazole plant protection fungicides, e.g., tebuconazole, triadimefon, triadimenol, paclobutrazol, and flutriafol. The two triazole ring isomers, 1,2,3-triazole and 1,2,4-triazole, are found in both plant protection fungicides and the others antifungal drugs, respectively (Carisse 2010).

Propiconazole, tebuconazole, and azaconazole are well known synthetic fungicides that are commonly used in commercial formulations for wood preservation 
against all types of wood-rotting fungi (Wüstenhöfer et al. 1993). However, 1,2,4triazoles have additional benefits of having a low toxicity to animals and being environmentally stable (Bakhsous et al. 2006). Propiconazole, tebuconazole, and chlorothalonile have shown inhibitory activity of $49.3 \%, 40.4 \%$, and $7.1 \%$, respectively, against the growth of T. versicolor fungus at $450 \mathrm{ppm}$ (Hosseinihashemi et al. 2016a, Nazari and Hosseinihashemi 2017a; Nazari and Hosseinihashemi 2017b).

Shi et al. (2014) studied the synthesis and in vitro activity of 1-((benzofuran-2yl)methyl)-1H triazole derivatives against a panel of five different human tumor cell lines and showed that benzotriazole, i.e., a 1,2,3-triazole ring with a substitution of the triazolyl-3-position with a naphthylacyl, 4-bromophenacyl, or 4-methylbenzyl group, could be crucial for promoting cytotoxic activity. Likewise, some of the studied compounds were found most potent with selectively against HL-60, SMMC-7721, and MCF-7 cell lines, respective to the above substitutions, and in particular, compound 20 was selective against the HL-60 and A549 cell lines with $\mathrm{IC}_{50}$ values of $0.62 \mu \mathrm{M}$ and $1.60 \mu \mathrm{M}$.

A series of benzofuran-triazole hybrids were designed and synthesized by Liang et al. (2016), and the in vitro antifungal activity of the final compounds were evaluated using the microdilution broth method against five strains of pathogenic fungi. This study indicated that the target compounds exhibited moderate to satisfactory activity.

Some representative 1,2,3-triazole derivatives with antifungal activity are shown in Fig. 1 (Liang et al. 2016).

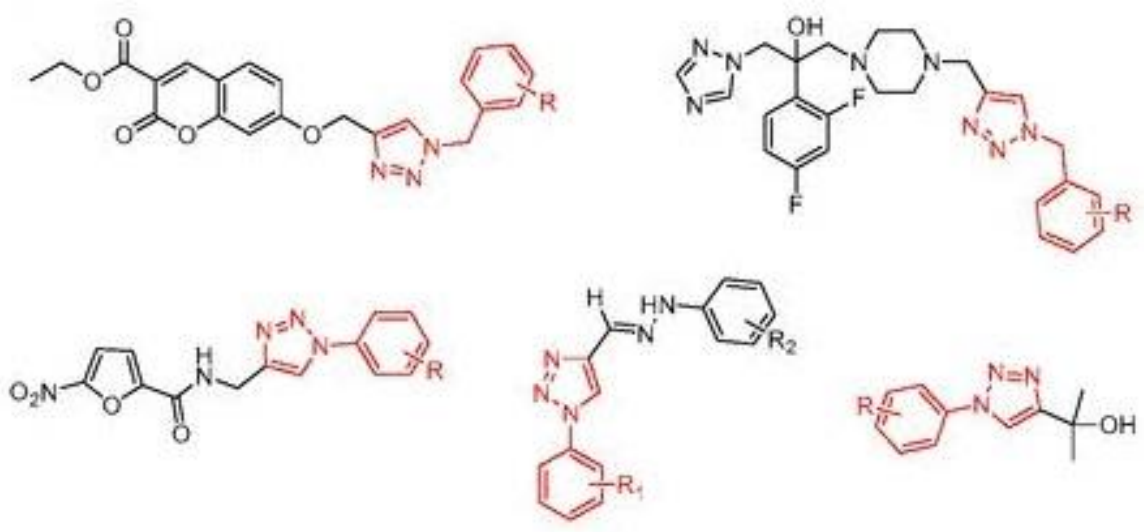

Fig. 1. Representative 1,2,3-triazole derivatives with antifungal activity

Recently, the 1,2,3-triazole scaffold became popular prominent fragment with the emergence of click chemistry. The 1,2,3-triazole-containing compounds have been reported to possess a variety of biological activities (Giffin et al. 2008; Weide et al. 2010; Xu et al. 2013; Kumar et al. 2014; Mohammadi-Khanaposhtani et al. 2015), especially as antifungal agents (Lima-Neto et al. 2012; Dai et al. 2015; Kamal et al. 2015). Furthermore, hybridizations of the 1,2,3-triazole moiety with other antifungal agents were reported (Giffin et al. 2008).

Reports by Hosseinihashemi et al. (2020), in regards to the effects of new benzofuran-1,3,4-oxadiazole hybrids on the inhibition of mycelial growth in wooddegrading fungi, showed that most of the compounds at $500 \mathrm{ppm}$ concentration did not exhibit acceptable antifungal effects, but they had better antifungal activity at $1000 \mathrm{ppm}$ concentration. Compounds 5a, 5c, and 5i at a concentration of $1000 \mathrm{ppm}$ showed 
inhibition percentages of $14.6 \%, 23.0 \%$, and $14.7 \%$, respectively, against the growth of $P$. placenta and $C$. puteana. Among the tested compounds, 2-(benzofuran-2-yl)-5-((2,6difluorobenzyl)thio)-1,3,4-oxadiazole (5h) hybrid was the most active one.

It is unclear whether such fungi ( $P$. placenta and $C$. puteana) have a generic ability to degrade pesticides, and whether similar degradative abilities are ubiquitous among white-rot fungi (Bending et al. 2002).

Triazole fungicides, e.g., propiconazole and tebuconazole, inhibit the C14 demethylation step in the fungal ergosterol biosynthesis as demethylation inhibitors (DMIs) and thereby interfere with the basic metabolism of the fungal cell walls and contents (Copping et al. 1984).

Encouraged by the above results, the authors attempted to design and synthesize a series of new benzofuran-1,2,3-triazole hybrids to evaluate the in vitro antifungal activity against wood-deterioration fungi.

\section{EXPERIMENTAL}

\section{Preparation of Synthesized Materials}

All chemical compounds were purchased from Merck (Darmstadt, Germany), Sigma-Aldrich (Darmstadt, Germany), and Acros Chemical (Schwerte, Germany) and used without further purification.

Synthesis of ethyl benzofuran-2-carboxylates (2)

A mixture of 2-hydroxybenzaldehydes (1) $(0.05 \mathrm{~mol})$, ethyl bromoacetate $(0.05 \mathrm{~mol})$, anhydrous potassium carbonate $(0.075 \mathrm{~mol})$, and dried dimethylformamide (DMF) $(70 \mathrm{~mL})$ was heated at $90{ }^{\circ} \mathrm{C}$ for $4 \mathrm{~h}$. This solution was decanted into ice water and the precipitate was filtered off and washed with cold water to afford ethyl benzofuran-2-carboxylate as cream powder (yield: $75 \%$; M. p. $32{ }^{\circ} \mathrm{C}$ ).

Synthesis of benzofuran-2-carboxylic acids (3)

Ethyl-1-benzofuran-2-carboxylate (2) $(1 \mathrm{mmol})$ containing ethanol was added to water (at a 2 to 1 ratio, $30 \mathrm{~mL}$ ) and potassium hydroxide $(2 \mathrm{mmol})$; this mixture was refluxed for $2 \mathrm{~h}$. The reaction was monitored via thin layer chromatography (TLC). After the reaction was completed, the reaction mixture was decanted into ice water and extracted with ethyl acetate. The ethyl acetate layer was separated, dried, and evaporated under vacuum to yield benzofuran-2-carboxylic acid (3) as a white crystal (yield: 85\%; M. p. $\left.190{ }^{\circ} \mathrm{C}\right)$.

\section{Synthesis of N-(prop-2-ynyl)benzofuran-2-carboxamides (5)}

A mixture of benzofuran-2-carboxylic acid (3) (1.5 mmol), HOBt (1.7 mmol), and EDC. $\mathrm{HCl}(1.7 \mathrm{mmol})$ in dried acetonitrile $(10 \mathrm{~mL})$ was stirred for $30 \mathrm{~min}$ at ambient temperature. Propargylamine $(1.8 \mathrm{mmol})$ was added and the mixture was stirred for $24 \mathrm{~h}$ to $48 \mathrm{~h}$. After the reaction was completed (checked via TLC), the crude product was extracted with chloroform, a citric acid solution (10\%), and sodium hydrogen carbonate $(10 \%)$. The organic layer was separated, dried, and evaporated under a vacuum to yield $\mathrm{N}$-(prop-2-ynyl)benzofuran-2-carboxamides as a cream powder (5) (yield: 78\%, M. p. $160.1-164{ }^{\circ} \mathrm{C}$. 
General procedure for the synthesis of product $\mathbf{8 a - g}$

Triethylamine $(1 \mathrm{mmol})$ and $\mathrm{H}_{2} \mathrm{O} / \mathrm{t}-\mathrm{BuOH}(1: 1)(5 \mathrm{~mL})$ were added to a mixture of benzyl bromide (6) $(1.3 \mathrm{mmol})$ and sodium azide $(1 \mathrm{mmol})$ and stirred for $1 \mathrm{~h}$ at room temperature. After that, $N$-(prop-2-yn-1-yl)benzofuran-2-carboxamide (5) (1 mmol), sodium ascorbate, and $\mathrm{CuSO}_{4} .5 \mathrm{H}_{2} \mathrm{O}(7 \mathrm{~mol} \%)$ were added to the reaction mixture and stirred at room temperature for $24 \mathrm{~h}$ to $48 \mathrm{~h}$. Then, the reaction mixture was diluted with cold water and poured into crushed ice and precipitated products $8 \mathbf{8}, \mathbf{8 b}, \mathbf{8 c}, \mathbf{8 d}, \mathbf{8 e}$, and $\mathbf{8 g}$ were filtered off, washed with water, and purified via recrystallization in ethanol.

The proposed procedure for the synthesis of designed compounds $\mathbf{8 a}$ through $\mathbf{8 g}$ is depicted in Fig. 2.

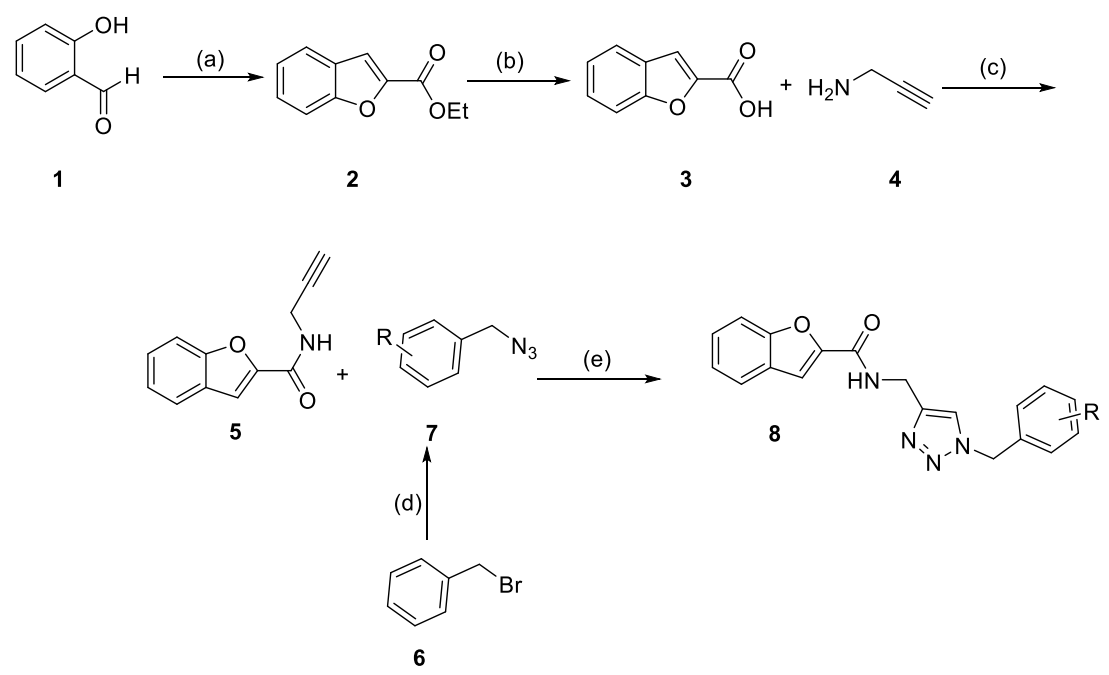

Fig. 2. Synthesis of benzofuran-1,2,3-triazole hybrids: (a) Ethyl bromoacetate, $\mathrm{K}_{2} \mathrm{CO}_{3}$, Dry DMF, $90^{\circ} \mathrm{C}, 4 \mathrm{~h}$ to $6 \mathrm{~h}$; (b) $\mathrm{KOH}$, Ethanol: $\mathrm{H}_{2} \mathrm{O}$ (2 to 1 ratio), reflux; (c) $\mathrm{HOBT}$, EDC. $\mathrm{HCl}$, dry $\mathrm{CH}_{3} \mathrm{CN}$, room temperature (r.t.), $24 \mathrm{~h}$ to $48 \mathrm{~h}$; (d) $\mathrm{NaN}_{3}, \mathrm{NEt}_{3}, \mathrm{H}_{2} \mathrm{O} / \mathrm{t}-\mathrm{BuOH}$ (1 to 1 ratio), 1 h, r.t.; and (e) CuSO $4.5 \mathrm{H}_{2} \mathrm{O}$, Sodium ascorbate, r.t., $24 \mathrm{~h}$ to $48 \mathrm{~h}$

N-((1-(2-methylbenzyl)-1H-1,2,3-triazol-4-yl)methyl)benzofuran-2-carboxamide (8a)

White powder; 0.298 g, Yield: $(86 \%)$; M. p. $147-150{ }^{\circ} \mathrm{C}$. IR $(\mathrm{KBr})\left(v_{\max } / \mathrm{cm}^{-1}\right)$ : $3421(\mathrm{NH}), 1659(\mathrm{CO}) .{ }^{1} \mathrm{H}$ NMR $\left(500 \mathrm{MHz} \mathrm{CDCl}_{3}\right): \delta 2.30(3 \mathrm{H}, \mathrm{s}, \mathrm{Me}), 4.75(2 \mathrm{H}, \mathrm{bs})$, $5.51(2 \mathrm{H}, \mathrm{s}), 7.15(1 \mathrm{H}, \mathrm{bs}), 7.21(2 \mathrm{H}, \mathrm{d}, J=6 \mathrm{~Hz}), 7.26$ to $7.28(2 \mathrm{H}, \mathrm{m}), 7.39(2 \mathrm{H}, \mathrm{t}, J=$ $6 \mathrm{~Hz}), 7.44$ to $7.47(2 \mathrm{H}, \mathrm{m}), 7.64(1 \mathrm{H}, \mathrm{d}, J=6 \mathrm{~Hz}) .{ }^{13} \mathrm{C}$ NMR $\left(125 \mathrm{MHz}, \mathrm{DMSO}-d_{6}\right): \delta$ $18.60,34.34$, 50.80, 109.44, 109.57, 111.73, 122.71, 123.05, 123.19, 123.71, 126.17, $126.85,128.25,128.69,130.35,136.27,144.79,148.88,154.17$, and 158.05. Combustion elemental analysis (Anal.) calculated (Calcd) for $\mathrm{C}_{20} \mathrm{H}_{18} \mathrm{~N}_{4} \mathrm{O}_{2}$ : C, 69.35; H, 5.24; N, 16.17. Found: C, 69.48; H, 5.51; N, 16.04.

$N$-((1-(2-chlorobenzyl)-1H-1,2,3-triazol-4-yl)methyl)benzofuran-2-carboxamide (8b)

White powder; 0.319 g, Yield: $(87 \%)$; M. p. $149-152{ }^{\circ} \mathrm{C}$. IR $(\mathrm{KBr})\left(v_{\max } / \mathrm{cm}^{-1}\right)$ : $3417(\mathrm{NH}), 1656(\mathrm{CO}) .{ }^{1} \mathrm{H} \mathrm{NMR}\left(500 \mathrm{MHz} \mathrm{CDCl}_{3}\right): \delta 4.79(2 \mathrm{H}, \mathrm{bs}), 5.67(2 \mathrm{H} \mathrm{s}), 7.20$ $(1 \mathrm{H}, \mathrm{bs}), 7.26$ to $7.33(3 \mathrm{H}, \mathrm{m}), 7.40(1 \mathrm{H}, \mathrm{s}), 7.43(1 \mathrm{H}, \mathrm{t}, J=7.5 \mathrm{~Hz}), 7.47(1 \mathrm{H}, \mathrm{d}, J=$ $5.8 \mathrm{~Hz}), 7.49(1 \mathrm{H}, \mathrm{s}), 7.80(1 \mathrm{H}, \mathrm{bs}, \mathrm{NH}-) .{ }^{13} \mathrm{C} \mathrm{NMR}\left(125 \mathrm{MHz}, \mathrm{DMSO}-d_{6}\right): \delta 34.69$, 51.43, 110.54, 111.78, 122.62, 123.64, 126.93, 127.43, 127.55, 129.92, 130.26, 130.36, 132.21, 144.63, 148.32, 154.72, and 158.84. EI-MS, $m / z: 366.2\left(\right.$ Calcd for $\mathrm{C}_{19} \mathrm{H}_{15} \mathrm{ClN}_{4} \mathrm{O}_{2}$ : 
366.81). Anal. Calcd for $\mathrm{C}_{19} \mathrm{H}_{15} \mathrm{ClN}_{4} \mathrm{O}_{2}$ : C, 62.21; H, 4.12; N, 15.27. Found: C, 62.13; $\mathrm{H}$, $4.02 ; \mathrm{N}, 15.46$.

N-((1-(3-methylbenzyl)-1H-1,2,3-triazol-4-yl)methyl)benzofuran-2-carboxamide (8c)

White powder; 0.283 g, Yield: $82 \%$; M. p. $174.5-176.3{ }^{\circ} \mathrm{C}$. IR $(\mathrm{KBr})\left(v_{\max } / \mathrm{cm}^{-1}\right)$ : $3420(\mathrm{NH}), 1655(\mathrm{CO}) .{ }^{1} \mathrm{H}$ NMR $\left(500 \mathrm{MHz}, \mathrm{CDCl}_{3}\right): \delta 2.32(3 \mathrm{H}, \mathrm{s}, \mathrm{Me}), 4.75(2 \mathrm{H}, \mathrm{bs})$, $5.46(2 \mathrm{H}, \mathrm{s}), 7.07$ to $7.09(2 \mathrm{H}, \mathrm{m}), 7.15(1 \mathrm{H}, \mathrm{d}, J=8 \mathrm{~Hz}), 7.23-7.28(2 \mathrm{H}, \mathrm{m}), 7.38(1 \mathrm{H}, \mathrm{t}$, $J=7.5 \mathrm{~Hz}), 7,44(1 \mathrm{H}, \mathrm{d}, J=6 \mathrm{~Hz}), 7.45(1 \mathrm{H}, \mathrm{s}), 7.58(1 \mathrm{H}, \mathrm{bs}, \mathrm{NH}-), 7.63(1 \mathrm{H}, \mathrm{d}, J=7.5$ $\mathrm{Hz}) .{ }^{13} \mathrm{C} \mathrm{NMR}\left(125 \mathrm{MHz}, \mathrm{CDCl}_{3}\right): \delta 21.28,34.80,54.32,110.50,111.82,122.63,123.67$, $125.23,126.93,127.49,128.88,129.00,120.55,134.36,138.96,148.47,154.80$, and 158.92. EI-MS, $m / z: 346.3$ (Calcd for $\mathrm{C}_{20} \mathrm{H}_{18} \mathrm{~N}_{4} \mathrm{O}_{2}$ : 346.3). Anal. Calcd for $\mathrm{C}_{20} \mathrm{H}_{18} \mathrm{~N}_{4} \mathrm{O}_{2}$ : C, 69.35; H, 5.24; N, 16.17. Found: C, 69.53; H, 5.11; N, 16.28.

$N$-((1-(3-methoxybenzyl)-1H-1,2,3-triazol-4-yl)methyl)benzofuran-2-carboxamide $(\mathbf{8 d})$

White powder; 0.309 g, Yield: 86\%; M. p. $127-128{ }^{\circ} \mathrm{C}$. IR $(\mathrm{KBr})\left(v_{\max } / \mathrm{cm}^{-1}\right)$ : $3462(\mathrm{NH}), 1657(\mathrm{CO}) .{ }^{1} \mathrm{H}$ NMR $\left(500 \mathrm{MHz}, \mathrm{CDCl}_{3}\right): \delta 3.73(3 \mathrm{H}, \mathrm{s},-\mathrm{OMe}), 4.71(2 \mathrm{H}, \mathrm{d}$, $J=5.3 \mathrm{~Hz}), 5.47(2 \mathrm{H}, \mathrm{s}), 6.78(1 \mathrm{H}, \mathrm{s}), 6.82$ to $6.85(2 \mathrm{H}, \mathrm{m}), 7.24(1 \mathrm{H}, \mathrm{t}, J=7.7 \mathrm{~Hz})$, $7.26(1 \mathrm{H}, \mathrm{s}), 7.36(1 \mathrm{H}, \mathrm{t}, J=7.7 \mathrm{~Hz}), 7.41(1 \mathrm{H}, \mathrm{d}, J=6.6 \mathrm{~Hz}), 7.42(1 \mathrm{H}, \mathrm{s}), 7.53(1 \mathrm{H}, \mathrm{bs}$, $\mathrm{NH}), 7.59$ to $7.62(2 \mathrm{H}, \mathrm{d}, J=8 \mathrm{~Hz}) .{ }^{13} \mathrm{C} \mathrm{NMR}\left(125 \mathrm{MHz}, \mathrm{CDCl}_{3}\right): \delta 34.68,54.27,55.24$, $110.57,111.79$, 113.74, 114.19, 120.29, 122.62, 123.64, 126.90, 126.94, 127.40, 130.18, 135.75, 148.28, 154.70, 158.85, and 160.02. EI-MS, $m / z: 362.3$ (Calcd for $\mathrm{C}_{20} \mathrm{H}_{18} \mathrm{~N}_{4} \mathrm{O}_{3}$ : 362.3). Anal. Calcd for $\mathrm{C}_{20} \mathrm{H}_{18} \mathrm{~N}_{4} \mathrm{O}_{3}$ : C, 66.29; H, 5.01; N, 15.46. Found: C, 66.37; H, 5.10; N, 15.39.

$N$-((1-(4-methoxybenzyl)-1H-1,2,3-triazol-4-yl)methyl)benzofuran-2-carboxamide $(\mathbf{8 e})$

White powder; 0.297 g, Yield: $81 \%$; M. p. $179-181{ }^{\circ} \mathrm{C}$. IR $(\mathrm{KBr})\left(v_{\max } / \mathrm{cm}^{-1}\right)$ : $\left.3457(\mathrm{NH}), 1656(\mathrm{CO}) .{ }^{1} \mathrm{H} \mathrm{NMR}\left(500 \mathrm{MHz}^{\mathrm{CDCl}}\right)_{3}\right): \delta 3.80(3 \mathrm{H}, \mathrm{s},-\mathrm{OMe}), 4.74(2 \mathrm{H}$, bs), $5.44(2 \mathrm{H}, \mathrm{s}), 6.88(2 \mathrm{H}, \mathrm{d}, J=8.5 \mathrm{~Hz}), 7.23(2 \mathrm{H}, \mathrm{d}, J=8.5 \mathrm{~Hz}), 7.26$ to $7.28(2 \mathrm{H}, \mathrm{m})$, $7.41(1 \mathrm{H}, \mathrm{t}, J=7.5 \mathrm{~Hz}), 7.45(1 \mathrm{H}, \mathrm{s}), 7.46(1 \mathrm{H}, \mathrm{d}, J=7.5 \mathrm{~Hz}), 7.60(1 \mathrm{H}, \mathrm{bs},-\mathrm{NH}), 7.65$ $(1 \mathrm{H}, \mathrm{d}, J=7.5 \mathrm{~Hz}) .{ }^{13} \mathrm{C}$ NMR $\left(125 \mathrm{MHz}, \mathrm{CDCl}_{3}\right): \delta 34.55,54.43,55.30,110.65,111.81$, $114.47,122.64,123.67,126.31,126.97,127.44,129.79,133.10,137.11,139.10,146.10$, 148.33, 154.76, 158.82, and 160.02. Anal. Calcd for $\mathrm{C}_{20} \mathrm{H}_{18} \mathrm{~N}_{4} \mathrm{O}_{3}: \mathrm{C}, 66.29 ; \mathrm{H}, 5.01 ; \mathrm{N}$, 15.46. Found: C, 66.18; H, 5.16; N, 15.25.

N-((1-(4-fluorobenzyl)-1H-1,2,3-triazol-4-yl)methyl)benzofuran-2-carboxamide (8f)

Pale yellow powder; 0.301 g, Yield: $86 \%$; M. p. $173-175^{\circ} \mathrm{C} .{ }^{1} \mathrm{H}$ NMR (500 MHz, $\left.\mathrm{CDCl}_{3}\right): \delta 4.70(2 \mathrm{H}, \mathrm{bs}), 5.48(2 \mathrm{H}, \mathrm{s}), 7.06(2 \mathrm{H}, \mathrm{t}, J=8 \mathrm{~Hz}), 7.26$ to $7.30(4 \mathrm{H}, \mathrm{m}), 7.41$ $(1 \mathrm{H}, \mathrm{m}), 7.46$ to $7.49(2 \mathrm{H}, \mathrm{m}), 7.66(1 \mathrm{H}, \mathrm{m}) .{ }^{13} \mathrm{C} \mathrm{NMR}\left(125 \mathrm{MHz}, \mathrm{CDCl}_{3}\right): \delta 34.39$, 54.43, 110.72, 111.80, 116.02, 116.19, 122.67, 123.71, and 127.01, 127.44, 130.08. Anal. Calcd for $\mathrm{C}_{19} \mathrm{H}_{15} \mathrm{FN}_{4} \mathrm{O}_{2}$ : C, 65.14; H, 4.32; N, 15.99. Found: C, 65.23; H, 4.21; N, 16.08.

N-((1-(4-bromobenzyl)-1H-1,2,3-triazol-4-yl)methyl)benzofuran-2-carboxamide (8g)

White powder; 0.370 g, Yield: 90\%; M. p. $197-199{ }^{\circ} \mathrm{C}$. IR $(\mathrm{KBr})\left(v_{\max } / \mathrm{cm}^{-1}\right)$ :

$3411(\mathrm{NH}), 1655(\mathrm{CO}) .{ }^{1} \mathrm{H}$ NMR $\left(500 \mathrm{MHz}, \mathrm{CDCl}_{3}\right): \delta 4.76(2 \mathrm{H}, \mathrm{bs}), 5.47(2 \mathrm{H}, \mathrm{s}), 7.15$ $(2 \mathrm{H}, \mathrm{d}, J=8 \mathrm{~Hz}), 7.26(1 \mathrm{H}, \mathrm{s}), 7.29(1 \mathrm{H}, \mathrm{t}, J=7.5 \mathrm{~Hz}), 7.42(2 \mathrm{H}, \mathrm{m}), 7.42(1 \mathrm{H}, \mathrm{t}, J=$ $7.5 \mathrm{~Hz}), 7.46$ to $7.51(4 \mathrm{H}, \mathrm{m}), 7.66(1 \mathrm{H}, \mathrm{d}, J=7.7 \mathrm{~Hz})$. EI-MS, m/z: 410.2 (Calcd for $\mathrm{C}_{19} \mathrm{H}_{15} \mathrm{BrN}_{4} \mathrm{O}_{2}$ : 411.2). Anal. Calcd for $\mathrm{C}_{19} \mathrm{H}_{15} \mathrm{BrN}_{4} \mathrm{O}_{2}$ : C, 55.49; H, 3.68; N, 13.62. Fund: C, 55.38; H, 3.79; N, 13.53. 


\section{Antifungal Activity Evaluation}

To evaluate the antifungal properties of various compounds, $12 \mathrm{mg}$ of each of the synthetic fungicides (dry powder) was dissolved into $12 \mathrm{~mL}$ of methanol $(\mathrm{MeOH})$ (Merck, Darmstadt, Germany), used as a solvent. The solution was passed by syringe from a $0.45 \mu \mathrm{m}$ Microsolve filter and poured into a sterile glass vial. Approximately 25 $\mathrm{mL}$ of the sterilized medium was placed in an autoclave for $20 \mathrm{~min}$ at $120{ }^{\circ} \mathrm{C}$ and 1.2 atm, and then it was poured into Petri plates. Then $25 \mu \mathrm{L}$ of the solution was added via micro-sampler at various concentrations $(500 \mathrm{ppm}$ and $1000 \mathrm{ppm}$ on four antibiogram discs) to medium containing malt extract agar (MEA) for microbiology (Merck KgaA 64271 Darmstadt, Germany), pH value: 5.6 (48 g/L, $\mathrm{H}_{2} \mathrm{O}, 22^{\circ} \mathrm{C}$ ) after autoclaving and were each poured into one of the Petri plates.

Ketoconazole, as the representative synthetic fungicide (at $500 \mathrm{ppm}$ and 1000 ppm concentrations), was used as the positive control due to its inhibitory effects against radial growth of three wood-degrading fungi, i.e., T. versicolor, $P$. placenta, and $C$. puteana. Methanol was selected as the negative control to confirm that there was no inhibitory effect (Teoh et al. 2015; Hosseinihashemi et al. 2020).

The plates were cooled in a sterile hood and inoculated with $0.50 \mathrm{~cm}$ plugs of Trametes versicolor, Poria placenta, Coniophora puteana, and Gloeophyllum trabeum fungi mycelia, which were introduced into the center of the Petri plate. The inoculated plates were incubated at $23{ }^{\circ} \mathrm{C}$ and $75 \%$ relative humidity without light. Four replicate antibiogram discs and one plate were used per treatment. The fungi were also grown on plates with non-compound MEA (i.e., with methanol) as a negative control. The percentage of fungal mycelial growth was plotted against the compound concentration, and the toxicity level was determined by the compound concentration. In other words, the fungal growth was monitored daily by measuring the percentage of area that was covered by fungus in the test plates until one day prior to complete coverage of Petri plate, similar to reported methods (Hosseinihashemi et al. 2016a, 2016b; Hosseinihashemi et al. 2020).

The colony fungal growth (shown by radius) was measured, and the percentage inhibition was calculated according to Eq 1.,

Percentage inhibition $=[(C-T) / C] \times 100$

where $C=\left[\frac{\left(C_{1}+C_{2}+C_{3}+C_{4}\right)}{4}\right]$ is the average colony radius (mm) of the negative control and $T=\left[\frac{\left(T_{1}+T_{2}+T_{3}+T_{4}\right)}{4}\right]$ is the average colony radius (mm) of the test plate.

\section{Chemistry Methods and Procedures}

The progress of the reaction was monitored via thin layer chromatography (silica gel 250 micron, F254 plates, Merck, Darmstadt, Germany). Melting points were determined using an Electrothermal 9100 apparatus. The IR spectra were recorded with a Shimadzu IR-460 spectrometer. The ${ }^{1} \mathrm{H}$ and ${ }^{13} \mathrm{C}$ NMR spectra were measured with a Bruker DRX-500 AVANCE (at 500.1 MHz and $125.8 \mathrm{MHz}$ ) instrument. The mass spectra were recorded with an Agilent Technology (HP) mass spectrometer, which operated at an ionization potential of $20 \mathrm{eV}$. The elemental analysis was performed with an ElementarAnalysen system GmbH VarioEL (CHN mode). 
Statistical analysis

Percentage inhibition was calculated and an analysis of variance for the different treatments was conducted using Duncan's test.

\section{RESULTS AND DISCUSSION}

\section{Chemistry}

Figure 2 describes the condensation reaction between salicylaldehyde (1) and ethyl bromoacetate in the presence of $\mathrm{K}_{2} \mathrm{CO}_{3}$, which afforded ethyl benzofuran-2carboxylates (2). The subsequent hydrolysis in aqueous ethanol/KOH yielded benzofuran-2-carboxylic acid (3). The propargylation of the latter compounds with propargyl amine (4) in the presence of hydroxybenzotriazole (HOBt) and $\mathrm{N}$ - $(3$ dimethylaminopropyl)- $N$ '-ethylcarbodiimide hydrochloride (EDC.HCl) as coupling agents resulted in compound (5). The $N$-(prop-2-ynyl)benzofuran-2-carboxamide (5) compounds were used for the click reaction. For this purpose, various benzyl bromide derivatives (6) and sodium azide, reacted in the presence of $\mathrm{Et}_{3} \mathrm{~N}$ in $\mathrm{H}_{2} \mathrm{O} / \mathrm{t}-\mathrm{BuOH}$ at room temperature. Then, $\mathrm{N}$-(prop-2-ynyl)benzofuran-2-carboxamide (5), $\mathrm{CuSO}_{4} .5 \mathrm{H}_{2} \mathrm{O}$, and sodium ascorbate were added to the freshly prepared azide derivatives (7) and the reaction was continued at room temperature for $8 \mathrm{~h}$ to $12 \mathrm{~h}$ to give the corresponding products $\mathbf{8 a}$ through $\mathbf{8 g}$ with sufficient yields, i.e., $80 \%$ to $90 \%$ (as shown in Fig. 3).
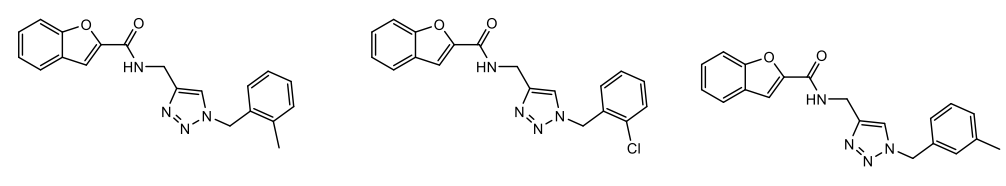

$8 \mathrm{a}(86 \%)$

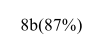

$8 \mathrm{c}(82 \%)$
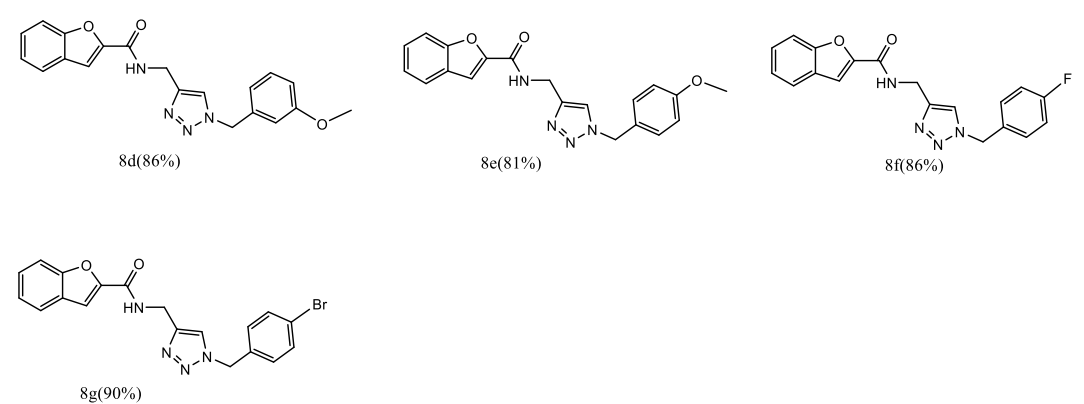

Fig. 3. Final compounds: $8 \mathrm{a}, \mathbf{8 b}, \mathbf{8 c}, \mathbf{8 d}, \mathbf{8 e}, \mathbf{8 f}$, and $\mathbf{8 g}$

\section{Activity Evaluation of Antifungal Compounds Against Fungi}

The in vitro antifungal activity of the target compounds was measured via the malt extract agar disk diffusion method with ketoconazole as the control fungicide or drug. The results are summarized in Table 1 and Fig. 4. The potential of synthetic compounds $8 \mathbf{a}, \mathbf{8 b}, \mathbf{8 c}, \mathbf{8 d}, \mathbf{8 e}, \mathbf{8 f}$, and $\mathbf{8 g}$ as wood preservatives were evaluated by determining the percentage of mycelial growth inhibition against the four tested wooddegrading fungi, Trametes versicolor, Poria placenta, Coniophora puteana, and Gloeophyllum trabeum, at $500 \mathrm{ppm}$ and $1000 \mathrm{ppm}$. Methanol and ketoconazole were used as a negative and positive control, respectively. 
Table 1. Mean \pm (Std.) Values of the \% Mycelial Growth Inhibition of Four Tested Wood-Degrading Fungi by the Seven Novel Synthetic Fungicide Preservatives and Ketoconazole at Two Concentrations per $25 \mathrm{~cm}^{3}$ of Malt Extract Agar (in One Day Prior to Complete Coverage by Fungus in the Negative Control Plates)

\begin{tabular}{|c|c|c|c|c|c|c|c|c|}
\hline Compound & \multicolumn{2}{|c|}{ T. versicolor } & \multicolumn{2}{|c|}{ P. placenta } & \multicolumn{2}{|c|}{ C. puteana } & \multicolumn{2}{|c|}{ G. trabeum } \\
\hline $8 \mathbf{a}$ & $\begin{array}{l}-3.57 a \\
(4.12)\end{array}$ & $\begin{array}{l}2.38 b \\
(2.75)\end{array}$ & $\begin{array}{l}0.00 a \\
(2.29)\end{array}$ & $\begin{array}{l}10.18 b \\
(1.39)\end{array}$ & $\begin{array}{l}1.14 a \\
(4.35)\end{array}$ & $\begin{array}{l}8.53 b \\
(9.16)\end{array}$ & $\begin{array}{l}1.71 a \\
(5.37)\end{array}$ & $\begin{array}{l}7.39 \mathrm{~b} \\
(2.17)\end{array}$ \\
\hline $8 b$ & $\begin{array}{c}0.00 \\
(5.14)\end{array}$ & $\begin{array}{l}5.95 \\
(3.07)\end{array}$ & $\begin{array}{l}-3.59 \\
(1.20)\end{array}$ & $\begin{array}{l}12.57 \\
(2.40)\end{array}$ & $\begin{array}{l}3.98 \\
(2.18)\end{array}$ & $\begin{array}{l}7.39 \\
(2.17)\end{array}$ & $\begin{array}{c}9.66 \\
(2.86)\end{array}$ & $\begin{array}{c}3.98 \\
(2.18)\end{array}$ \\
\hline 8c & $\begin{array}{c}1.19 \\
(1.37)\end{array}$ & $\begin{array}{l}10.71 \\
(3.08)\end{array}$ & $\begin{array}{l}-0.60 \\
(1.96)\end{array}$ & $\begin{array}{c}4.79 \\
(2.29)\end{array}$ & $\begin{array}{l}10.23 \\
(6.02)\end{array}$ & $\begin{array}{l}-1.72 \\
(1.32)\end{array}$ & $\begin{array}{l}15.34 \\
(2.17)\end{array}$ & $\begin{array}{c}4.55 \\
(1.86)\end{array}$ \\
\hline $8 d$ & $\begin{array}{l}2.38 \\
(1.94)\end{array}$ & $\begin{array}{c}4.76 \\
(0.00)\end{array}$ & $\begin{array}{l}-1.80 \\
(2.30)\end{array}$ & $\begin{array}{l}10.18 \\
(3.09)\end{array}$ & $\begin{array}{l}1.71 \\
(7.96)\end{array}$ & $\begin{array}{l}-1.15 \\
(3.43)\end{array}$ & $\begin{array}{c}3.41 \\
(1.32)\end{array}$ & $\begin{array}{l}28.22 \\
(6.85)\end{array}$ \\
\hline $8 e$ & $\begin{array}{c}2.98 \\
(1.19)\end{array}$ & $\begin{array}{l}2.98 \\
(2.99)\end{array}$ & $\begin{array}{l}-4.79 \\
(2.29)\end{array}$ & $\begin{array}{c}2.99 \\
(4.59)\end{array}$ & $\begin{array}{l}-1.14 \\
(1.31)\end{array}$ & $\begin{array}{l}11.93 \\
(6.78)\end{array}$ & $\begin{array}{c}1.71 \\
(2.86)\end{array}$ & $\begin{array}{l}13.07 \\
(5.68)\end{array}$ \\
\hline $8 f$ & $\begin{array}{c}1.79 \\
(1.19)\end{array}$ & $\begin{array}{l}-0.60 \\
(2.99)\end{array}$ & $\begin{array}{l}-4.19 \\
(1.39)\end{array}$ & $\begin{array}{c}0.00 \\
(1.20)\end{array}$ & $\begin{array}{l}23.87 \\
(3.94)\end{array}$ & $\begin{array}{c}6.82 \\
(4.91)\end{array}$ & $\begin{array}{l}47.16 \\
(3.41)\end{array}$ & $\begin{array}{l}15.91 \\
(0.00)\end{array}$ \\
\hline $8 g$ & $\begin{array}{l}-4.17 \\
(3.57)\end{array}$ & $\begin{array}{c}2.38 \\
(5.50) \\
\end{array}$ & $\begin{array}{c}0.00 \\
(1.20)\end{array}$ & $\begin{array}{c}0.60 \\
(1.39)\end{array}$ & $\begin{array}{c}5.12 \\
(9.16) \\
\end{array}$ & $\begin{array}{l}11.36 \\
(6.43) \\
\end{array}$ & $\begin{array}{c}3.98 \\
(2.18) \\
\end{array}$ & $\begin{array}{l}11.93 \\
(3.88) \\
\end{array}$ \\
\hline Descriptions & \multicolumn{8}{|c|}{ A: Mean \pm (std.) values at 500 ppm; b: Mean \pm (std.) values at $1000 \mathrm{ppm}$} \\
\hline $\begin{array}{c}\mathrm{C}(+) 500 \text { and } \\
1000(\mathrm{ppm})\end{array}$ & $\begin{array}{c}16.67 \mathrm{c} \\
(3.89)\end{array}$ & $\begin{array}{l}23.81 d \\
(2.75)\end{array}$ & $\begin{array}{l}2.40 c \\
(1.20)\end{array}$ & $\begin{array}{l}6.59 d \\
(3.39)\end{array}$ & $\begin{array}{l}12.50 \mathrm{c} \\
(7.06)\end{array}$ & $\begin{array}{l}17.62 d \\
(7.51)\end{array}$ & $\begin{array}{l}64.78 \mathrm{c} \\
(2.27)\end{array}$ & $\begin{array}{l}\text { 65.34d } \\
(5.68)\end{array}$ \\
\hline $\begin{array}{c}C(+) 30 \\
\text { and } 60(\mathrm{ppm})\end{array}$ & $\begin{array}{l}14.03 \\
(2.23)\end{array}$ & $\begin{array}{l}12.82 \\
(2.09)\end{array}$ & $\begin{array}{c}6.37 \\
(4.35)\end{array}$ & $\begin{array}{l}25.28 \\
(2.83)\end{array}$ & $\begin{array}{l}-26.87 \\
(6.22)\end{array}$ & $\begin{array}{l}2.26 \\
(7.91)\end{array}$ & $\begin{array}{l}14.63 \\
(5.97)\end{array}$ & $\begin{array}{l}29.27 \\
(1.99)\end{array}$ \\
\hline Descr & \multicolumn{8}{|c|}{$\begin{array}{l}\text { C: Mean } \pm \text { (std.) values at } 30 \mathrm{ppm} \text { and } 500 \mathrm{ppm} \\
\text { d: Mean } \pm \text { (std.) values at } 60 \mathrm{ppm} \text { and } 1000 \mathrm{ppm}\end{array}$} \\
\hline
\end{tabular}

8a: N-((1-(2-methylbenzyl)-1H-1,2,3-triazol-4-yl)methyl)benzofuran-2-carboxamide; 8b: N-((1-(2chlorobenzyl)-1H-1,2,3-triazol-4-yl)methyl)benzofuran-2-carboxamide; 8c: N-((1-(3-methylbenzyl)1H-1,2,3-triazol-4-yl)methyl)benzofuran-2-carboxamide; 8d: $\mathrm{N}$-((1-(3-methoxybenzyl)-1H-1,2,3triazol-4-yl)methyl)benzofuran-2-carboxamide; 8e: $\mathrm{N}$-((1-(4-methoxybenzyl)-1H-1,2,3-triazol-4yl)methyl)benzofuran-2-carboxamide; 8f: $\mathrm{N}$-((1-(4-fluorobenzyl)-1H-1,2,3-triazol-4yl)methyl)benzofuran-2-carboxamide; 8g: N-((1-(4-bromobenzyl)-1H-1,2,3-triazol-4yl)methyl)benzofuran-2-carboxamide; $\mathrm{C}(+)$ : Ketoconazole

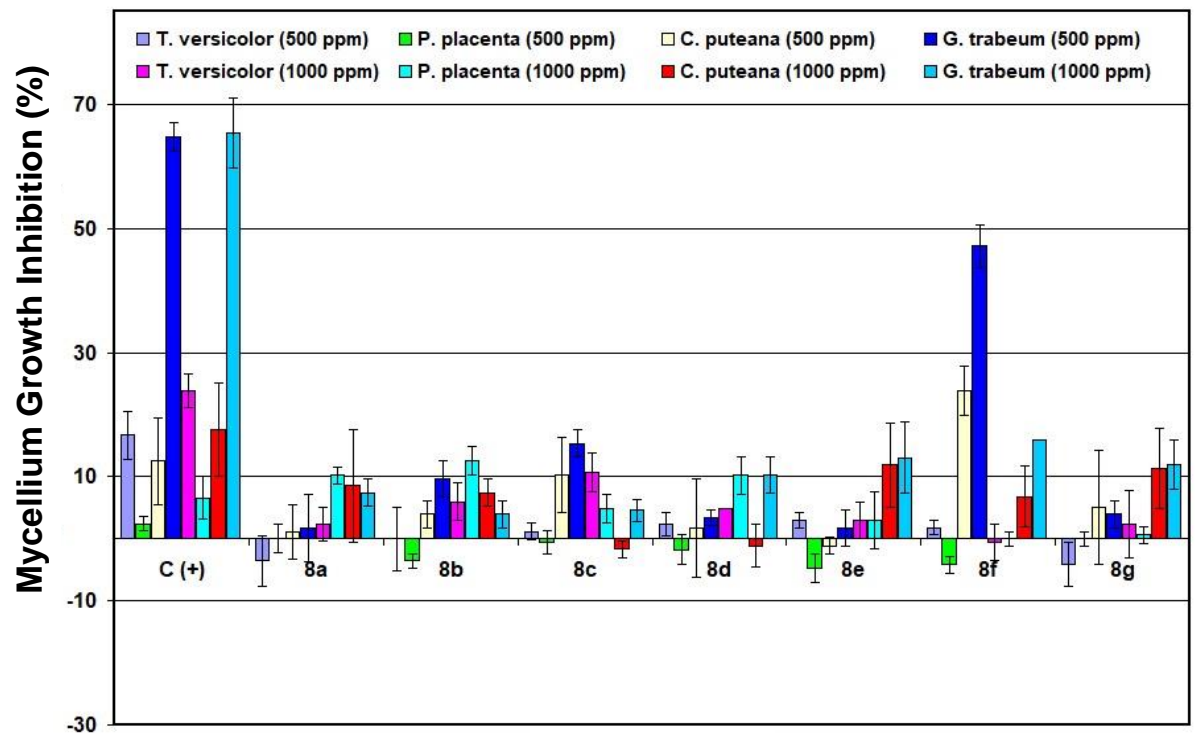

Compound

Fig. 4. Percentage of mycelial growth inhibited by synthetıc fungicides 
Statistically, there was a significant difference between the compounds and their concentrations on the percentage of mycelial growth inhibition of wood-degrading fungi (Table 2).

Table 2. Univariate Test Results for Percentage of Mycelial Growth Inhibition of the Four Tested Wood-Degrading Fungi by the Seven Novel Synthetic Fungicide Preservatives and Ketoconazole at Two Concentrations per $25 \mathrm{~cm}^{3}$ of Malt Extract Agar (in One Day Prior to Complete Coverage by Fungus in the Negative Control Plates)

\begin{tabular}{|c|c|c|c|c|c|}
\hline Source & $\begin{array}{c}\text { Type III Sum } \\
\text { of Squares }\end{array}$ & $\mathrm{df}$ & Mean Square & $\mathrm{F}$ & Sig. \\
\hline Corrected Model & 45951.662 & 63 & 729.391 & 20.170 & 0.000 \\
\hline Intercept & 16982.814 & 1 & 16982.814 & 469.627 & 0.000 \\
\hline Fungi (F) & 10251.709 & 3 & 3417.236 & 94.497 & 0.000 \\
\hline Concentrations (Con) & 645.382 & 1 & 645.382 & 17.847 & 0.000 \\
\hline Compounds (Com) & 13360.626 & 7 & 1908.661 & 52.780 & 0.000 \\
\hline $\mathrm{F}^{*}$ Con & 581.503 & 3 & 193.834 & 5.360 & 0.001 \\
\hline $\mathrm{F}^{*}$ Com & 15120.924 & 21 & 720.044 & 19.911 & 0.000 \\
\hline Con ${ }^{*}$ Com & 2639.349 & 7 & 377.050 & 10.427 & 0.000 \\
\hline $\mathrm{F}^{*}$ Con ${ }^{*}$ Com & 3352.168 & 21 & 159.627 & 4.414 & 0.000 \\
\hline Error & 6943.166 & 192 & 36.162 & & \\
\hline Total & 69877.642 & 256 & & & \\
\hline Corrected Total & 52894.828 & 255 & & & \\
\hline
\end{tabular}

According to the results, some of the target compounds exhibited acceptable antifungal activity at $500 \mathrm{ppm}$ and $1000 \mathrm{ppm}$. Compound 8c showed better antifungal activity at $1000 \mathrm{ppm}$ against T. versicolor (with a percentage inhibition of $10.71 \%$ ) in comparison to the other compounds with the exception of the positive control at all concentrations. In addition, compounds $\mathbf{8 b}, \mathbf{8 a}$, and $\mathbf{8 d}$ showed inhibition rates of $12.57 \%, 10.18 \%$, and $10.18 \%$, respectively, against $P$. placenta, which were more effective than the ketoconazole at $30 \mathrm{ppm}, 500 \mathrm{ppm}$, and $1000 \mathrm{ppm}$. However, ketoconazole showed better antifungal activity at $60 \mathrm{ppm}$ against $P$. placenta with a inhibition rate of $25.28 \%$. At $1000 \mathrm{ppm}$, better antifungal activity against $T$. versicolor and $P$. placenta was observed for most of the synthetic compounds. Particularly, compounds 8c, $\mathbf{8 f}, \mathbf{8 e}$, and $\mathbf{8 g}$ exhibited inhibition rates of $10.23 \%, 17.61 \%, 11.93 \%$, and $11.36 \%$, respectively, against the growth of $C$. puteana fungus. The greatest antifungal activities were for compound $\mathbf{8 c}$ at $500 \mathrm{ppm}$ and compound $8 \mathbf{f}$ at $500 \mathrm{ppm}$ and $1000 \mathrm{ppm}$ showing inhibition of $15.34 \%, 47.16 \%$, and $15.91 \%$, respectively, against G. trabeum. These two compounds were more effective than ketoconazol at $30 \mathrm{ppm}$ and $60 \mathrm{ppm}$. However, ketoconazole showed better antifungal activity at $500 \mathrm{ppm}$ and $1000 \mathrm{ppm}$ against $G$. trabeum fungus with an inhibition rate of $64.78 \%$ and $65.34 \%$, respectively.

The synthetic 1,2,3-triazole derivatives showed moderate control over the growth of fungi rather than their positive control for ketoconazole, at $30 \mathrm{ppm}, 60 \mathrm{ppm}, 500 \mathrm{ppm}$, and $1000 \mathrm{ppm}$ concentrations. Among all the novel synthetic compounds, N-((1-(4fluorobenzyl)-1H-1,2,3-triazol-4-yl)methyl)benzofuran-2-carboxamide (8f) was the most active antifungal agent against the mycelium growth of $G$. trabeum fungus.

The trends of the percentage inhibition of white-rot (T. versicolor) growth after 6 $\mathrm{d}$ (one day prior to complete coverage by fungus in the negative control plates) were shown in Fig. 5. As shown in Fig. 5, the compounds with greater and lower than 10\% 
inhibition are indicated by solid and dashed lines, respectively. Among the synthesized compounds that inhibited the growth of $T$. versicolor, $\mathrm{N}-((1-(4-m e t h o x y b e n z y l)-1 \mathrm{H}-$ 1,2,3-triazol-4-yl)methyl)benzofuran-2-carboxamide $(\mathbf{8 c})$ had the greatest performance, with an inhibition rate of $10.71 \%$ at a 1000 ppm concentration.

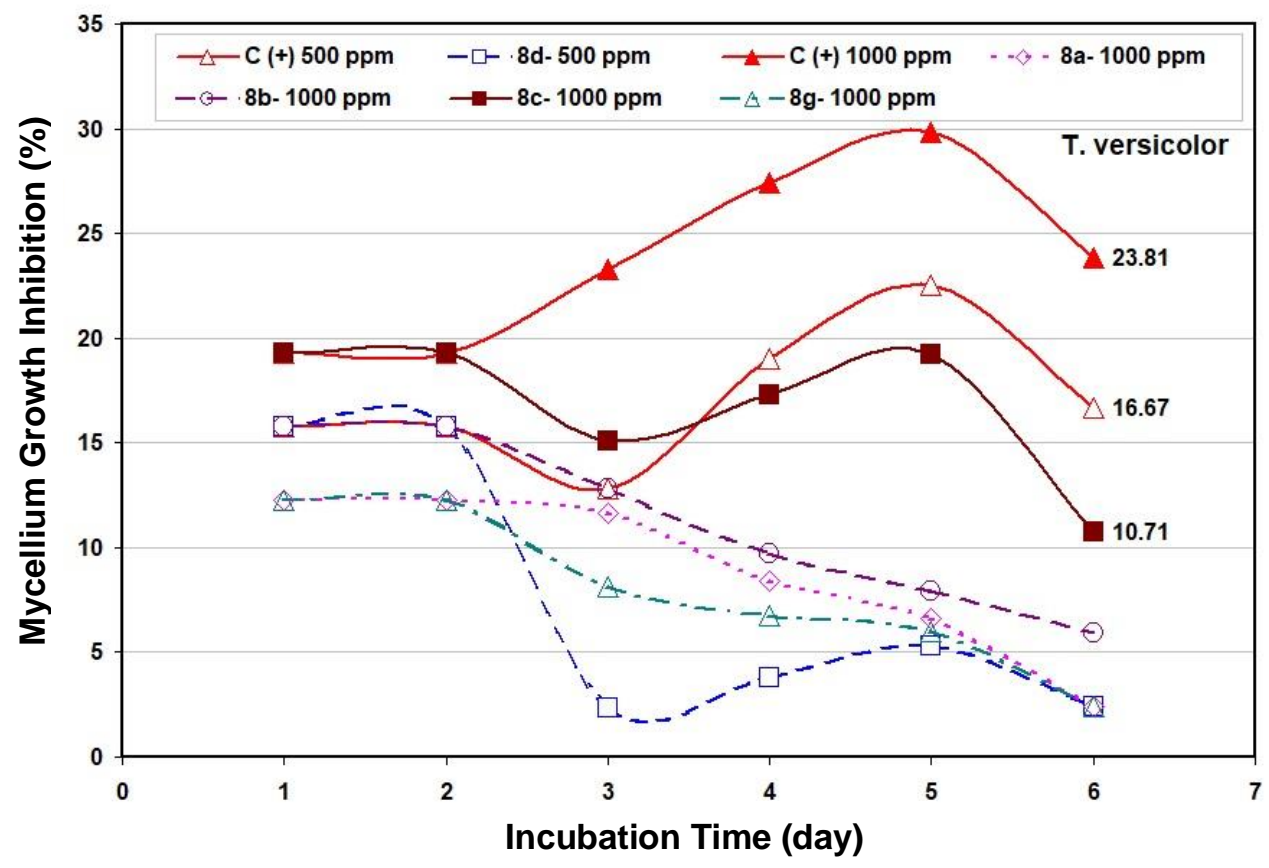

Fig. 5. Trends of the percentage inhibition of $T$. versicolor growth by different synthetic fungicides solutions with $500 \mathrm{ppm}$ and $1000 \mathrm{ppm}$ concentrations in $6 \mathrm{~d}$ (the $7^{\text {th }} \mathrm{d}$ was the final time)

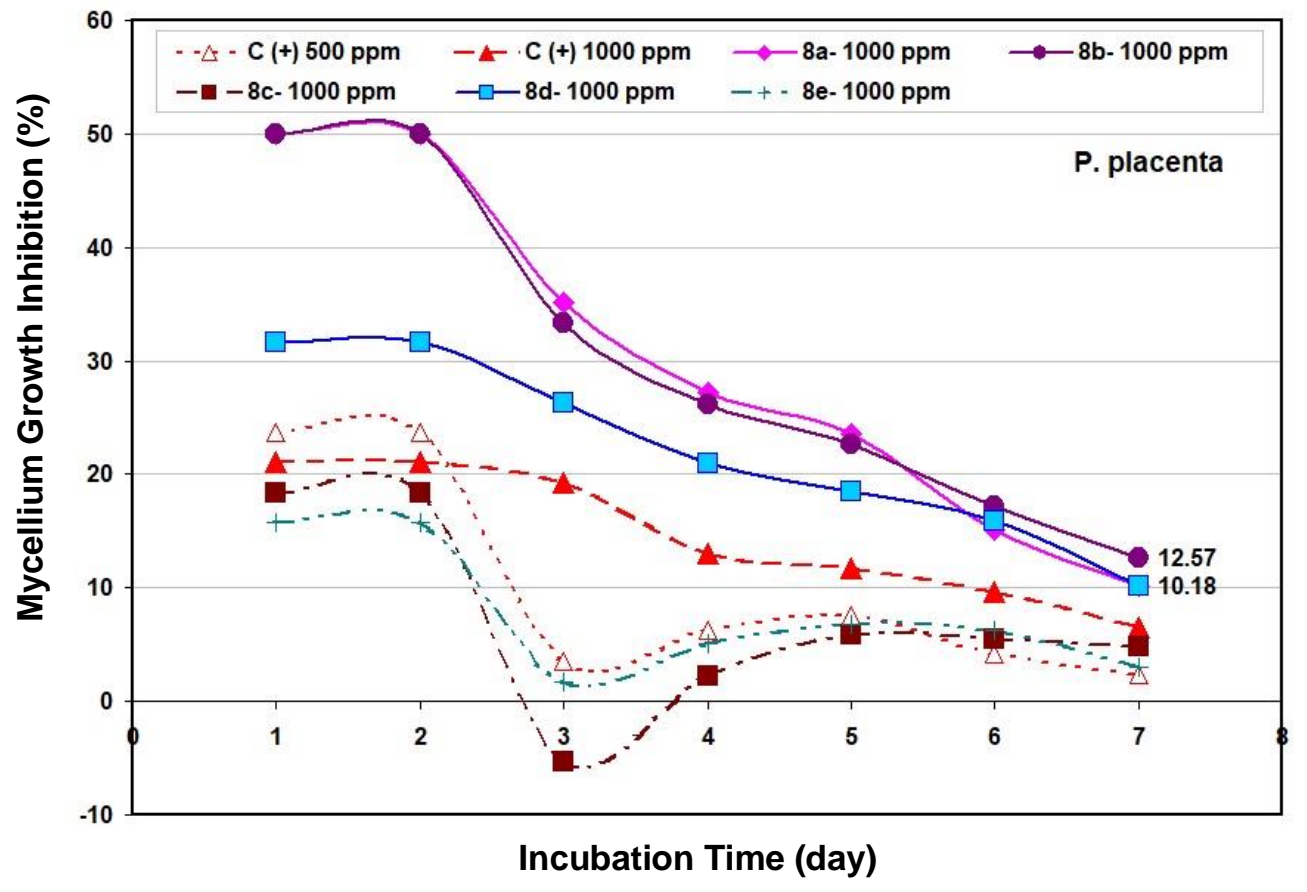

Fig. 6. Trends of the percentage inhibition of $P$. placenta growth by different synthetic fungicides solutions with $500 \mathrm{ppm}$ and $1000 \mathrm{ppm}$ concentrations in $7 \mathrm{~d}$ (the $8^{\text {th }} \mathrm{d}$ was the final time) 
Figure 6 illustrates the trends of percentage inhibition of dry brown-rot $(P$. placenta) growth after $7 \mathrm{~d}$ (one day prior to complete coverage by fungus in the negative control plates). In Fig. 6, the compounds above $0 \%$ to $10 \%$ of inhibition and above $10 \%$ of inhibition, were indicated by dashed and solid lines, respectively.

The most effective synthesized compounds that inhibited the growth of $P$. placenta were $\mathrm{N}$-((1-(2-chlorobenzyl)-1H-1,2,3-triazol-4-yl)methyl)benzofuran-2carboxamide $(\mathbf{8 b})$, with the position of 2-Cl substitution, $\mathrm{N}-((1-(2-$-methylbenzyl)-1H1,2,3-triazol-4-yl)methyl)benzofuran-2-carboxamide (8a), with the position of 2- $\mathrm{CH}_{3}$ substitution, and $\mathrm{N}-((1-(3-\mathrm{methoxybenzyl})-1 \mathrm{H}-1,2,3$-triazol-4-yl)methyl)benzofuran-2carboxamide $(\mathbf{8 d})$, with the position of $3-\mathrm{OCH}_{3}$ substitution, at a concentration of 1000 ppm $(12.57 \%, 10.18 \%$, and $10.18 \%$, respectively).

The trends of percentage inhibition of wet brown-rot (C. puteana) growth after 18 $\mathrm{d}$ (one day prior to complete coverage by fungus in the negative control plates) are shown in Fig. 7. As shown in Fig. 7, the compounds that had greater than $10 \%$ inhibition are indicated by solid lines. Among the novel synthesized compounds, N-((1-(4fluorobenzyl)-1H-1,2,3-triazol-4-yl)methyl)benzofuran-2-carboxamide $\quad(\mathbf{8 f})$ at a concentration of $500 \mathrm{ppm}$ could inhibited the growth of $C$. puteana by $23.86 \%$, which more effective than ketoconazole at the four various concentrations.

Figure 8 shows the trends of percentage inhibition of wet brown-rot (G. trabeum) growth after $11 \mathrm{~d}$ (one day prior to complete coverage by fungus in the negative control plates). As shown in Fig. 8, the compounds that had greater than 10\% inhibition were indicated by solid lines. Three synthesized compounds showed high growth percentage inhibition amounts $(15.34 \%, 15.91 \%$, and $47.16 \%)$, with the compound with the highest inhibition value was $\mathrm{N}-((1-(4-f l u o r o b e n z y l)-1 \mathrm{H}-1,2,3$-triazol-4-yl)methyl)benzofuran-2carboxamide (8f), at 500 ppm concentration.

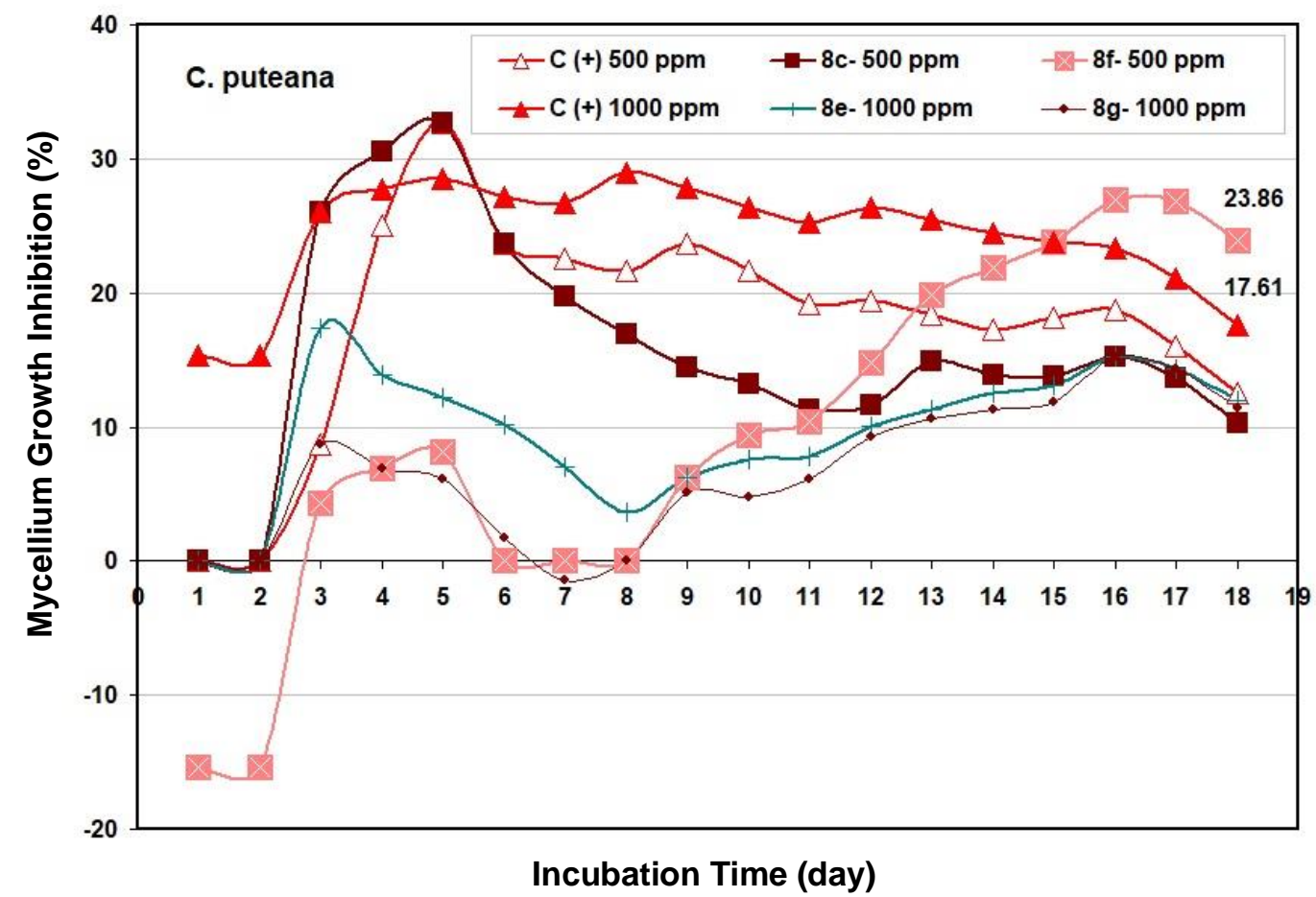

Fig. 7. Trends of the percentage inhibition of $C$. puteana growth by different synthetic fungicides solutions with $500 \mathrm{ppm}$ and $1000 \mathrm{ppm}$ concentrations in $18 \mathrm{~d}$ (the $19^{\text {th }} \mathrm{d}$ was the final time) 


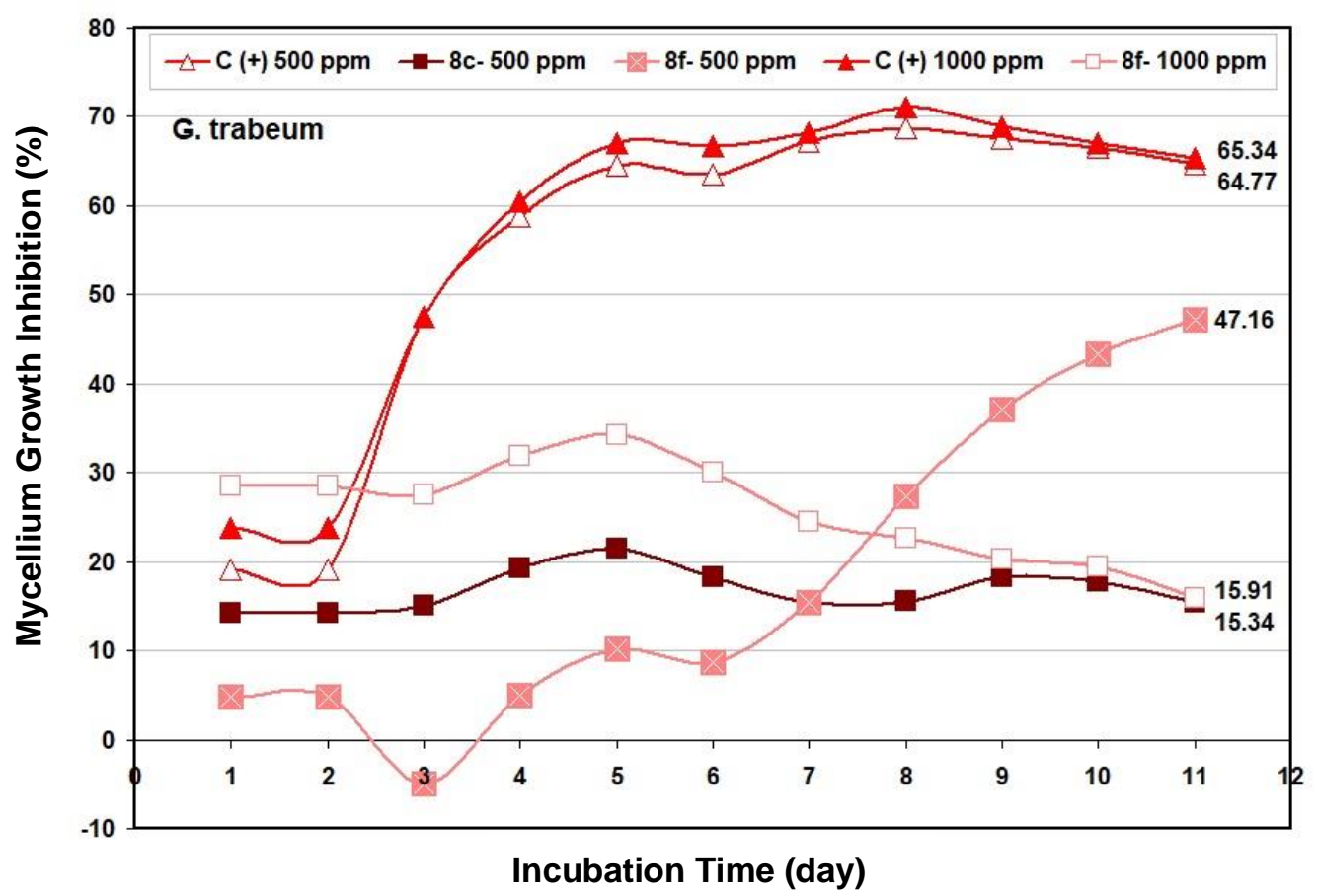

Fig. 8. Trends of the percentage inhibition of $G$. trabeum growth by different synthetic fungicides solutions with $500 \mathrm{ppm}$ and $1000 \mathrm{ppm}$ concentrations in $11 \mathrm{~d}$ (the $12^{\text {th }} \mathrm{d}$ was the final time)

As shown in Fig. 8 and Fig. 9b, the growth of G. trabeum stopped after $6 \mathrm{~d}$ when tested with compound $\mathbf{8 f}$, which at the final day of growth $\left(12^{\text {th }} \mathrm{d}\right)$ reached a percentage of inhibition of $48.33 \%$.
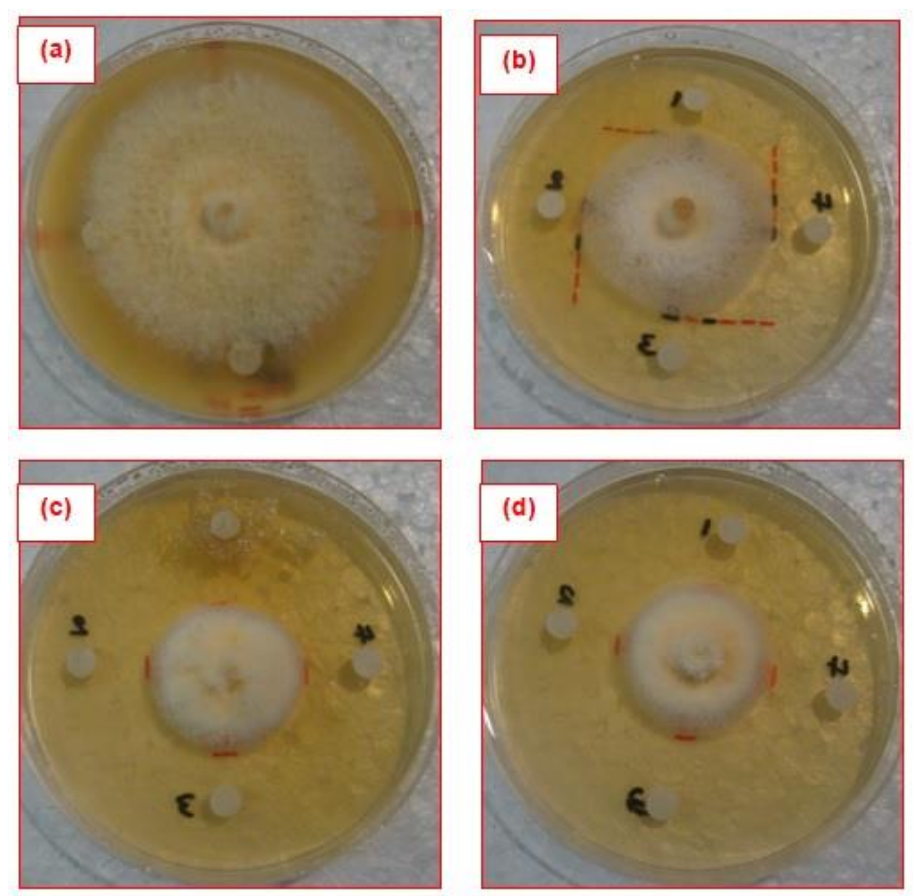

Fig. 9. Mycelial growth areas of the G. trabeum fungus; a: Negative control (44 mm); b: $8 \mathbf{f}$ at 500 ppm (23.25 mm); c: C (+) at 1000 ppm (15.25 mm); d: C (+) at 500 ppm (15.50 mm) 
Researchers have described both the biotransformation and biodegradation of some triazoles, such as tebuconazole and propiconazole, by the surrounding microbial species (fungi or bacteria) in liquid media (Obanda 2008; Obanda et al. 2008; Obanda and Shupe 2009), soil (Potter et al. 2005; White et al. 2010), and wood matrixes (Woo et al. 2010). Cleavage of the triazole ring is one of the major pathways of tebuconazole degradation by bacteria, mold, and soft and brown-rot fungi (Obanda 2008; Obanda and Shupe 2009). The hydroxyl group can be further acetylated or oxidized to form an ester or carboxyl group. Acetylation causes the deactivation of tebuconazole by decreasing its hydrophilicity (Kukowski 2018).

The presence of a methyl group provided similar activity to ketoconazole against $T$. versicolor and $P$. placenta at $1000 \mathrm{ppm}$ (Hosseinihashemi et al. 2020). A strong electron-donating group (methoxy group) with a meta position resulted in better activity against $P$. placenta than a para position at $1000 \mathrm{ppm}$. When the authors changed the position of the $-F$ substitution (strong electron-donating group) in the new benzofuran1,2,3-triazole hybride synthetic fungicide from the previous benzofuran-1,3,4-oxadiazole hybrid synthetic fungicide $5 \mathrm{~h}$ (meta to para position) led to more a potent compound $\mathbf{8 f}$ at 500 ppm against $C$. puteana and specially G. trabeum (Hosseinihashemi et al. 2020). However, this effect was not observed at $1000 \mathrm{ppm}$, which could be related to the low solubility of the compound at this concentration in the MEA medium. In compound $\mathbf{8 g}$, the presence of bromine in the para position, thus making a less electronegative atom, resulted in lower inhibition of the growth of T. versicolor and C. puteana fungi at 1000 ppm.

\section{CONCLUSIONS}

The synthesis and evaluation of new benzofuran-1,2,3-triazole hybrids as antifungal agents were investigated. Seven compounds (8a to 8g), for use as wood preservatives, were evaluated against white- and brown-rot fungi ( $T$. versicolor, $P$. placenta, C. puteana, and G. trabeum) at different concentrations (500 ppm and 1000 ppm) using in vitro disk diffusion.

1. The synthesized compounds with triazole linkage at $500 \mathrm{ppm}$ concentration may advance further development of new wood preservatives against the two wood brown-rotting fungi.

2. Compound $\mathbf{8 f}$ showed the highest antifungal activity against $C$. puteana and $G$. trabeum at $500 \mathrm{ppm}$ with a mycelial growth inhibition rate of $23.86 \%$ and $47.16 \%$, respectively.

3. Among the novel synthesized compounds, the complete inhibition of mycelial growth of $G$. trabeum fungus has been occurred in $6^{\text {th }} \mathrm{d}$ of incubation time only by $\mathbf{8 f}$ at a concentration of $500 \mathrm{ppm}$.

\section{ACKNOWLEDGMENTS}

The authors are grateful for the support of the Department of Medicinal Chemistry, Faculty of Pharmacy, Tehran University of Medical Sciences, Tehran, Iran 
and also the Department of Wood Science and Paper Technology, Karaj Branch, Islamic Azad University, Karaj, Iran.

\section{REFERENCES CITED}

Bakhsous, B., Dumarcay, S., Gelhaye, E., and Gérardin, P. (2006). Investigation of New Wood Preservation Formulations Based on Synergies Between Antioxidant, 2-HPNO and Propiconazole (IRG/WP 06-30401), International Research Group on Wood Protection, Troms $\varnothing$, Norway.

Bauschhaus, H. U., and Valcke, A. R. (1995). Triazoles: Synergism Between Propiconazole and Tebuconazole (IRG/WP 95-30092), International Research Group on Wood Protection, Helsingør, Denmark.

Bending, G. D., Friloux, M., and Walker, A. (2002). "Degradation of contrasting pesticides by white rot fungi and its relationship with ligninolytic potential," FEMS Microbiology Letters 212(1), 59-63. DOI:10.1016/S0378-1097(02)00710-3

Bruns, R., Kaulen, J., Kretschik, O., Kugler, M., and Uhr, H. (2005). "R\&D in material protection: New biocides," in: Directory of Microbicides for the Protection of Materials, W. Paulus (ed.), Springer, Dordrecht, Netherlands. DOI: 10.1007/1-40202818-0

Carisse, O. (2010). Fungicides, InTech, London, UK.

Christen, V., Crettaz, P., and Fent, K. (2014). "Additive and synergistic antiandrogenic activities of mixtures of azole fungicides and vinclozolin," Toxicology and Applied Pharmacology 279(3), 455-466. DOI: 10.1016/j.taap.2014.06.025

Copping, L. G., Birchmore, R. J., Wright, K., and Godson, D. H. (1984). "Structureactivity relationships in a group of imidazole-1-carboxamides," Pesticide Science 15(3), 280-284. DOI: 10.1002/ps.2780150310

Dai, Z.-C., Chen, Y.-F., Zhang, M., Li, S.-K., Yang, T.-T., Shen, L., Wang, J.-X., Qian, S.-S., Zhu, H.-L., and Ye, Y.-H. (2015). "Synthesis and antifungal activity of 1,2,3triazole phenylhydrazone derivatives," Organic \& Biomolecular Chemistry 13(2), 477-486. DOI: 10.1039/c4ob01758g

Giffin, M. J., Heaslet, H., Brik, A., Lin, Y.-C., Cauvi, G., Wong, C.-H., McRee, D. E., Elder, J. H., Stout, C. D., and Torbett, B. E. (2008). "A copper(I)-catalyzed 1,2,3triazole azide-alkyne click compound is a potent inhibitor of a multidrug-resistant HIV-1 protease variant," Journal of Medical Chemistry 51(20), 6263-6270. DOI: $10.1021 / \mathrm{jm} 800149 \mathrm{~m}$

Highley, T. (1976). "Hemicelluloses of white- and brown-rot fungi in relation to host preferences," Materials and Organisms 11(1), 33-46.

Hosseinihashemi, S. K., Nazari, L., Lashgari, A., and Salem, M. Z. M. (2016a).

"Evaluation of inner bark extract of barberry stem and its synergy with propiconazole, EDTA, BHT, and their combination against the white-rot fungus Trametes versicolor," BioResources 11(1), 1505-1517. DOI:

10.15376/biores.11.1.1505-1517

Hosseinihashemi, S. K., HosseinAshrafi, S. K., Jalali Goldeh, A., and Salem, M. Z. M. (2016b). "Antifungal and antioxidant activities of heartwood, bark, and leaf extracts of Robinia pseudoacacia,” BioResources 11(1), 1634-1646. DOI:

10.15376/biores.11.1.1634-1646

Hosseinihashemi, S. K., Toolabi, M., Abedinifar, F., Moghimi, S., Jalaligoldeh, A., 
Paknejad, F., Arabahmadi, S., and Foroumadi, A. (2020). "Synthesis and activity evaluation of new benzofuran-1,3,4-oxadiazole hybrids against wood-degrading fungi," BioResources 15(1), 1085-1097. DOI: 10.15376/biores.15.1.1085-1097

Kamal, A., Hussaini, S. M. A., Sucharitha, M. L., Poornachandra, Y., Sultana, F., Ganesh and Kumar, C. (2015). "Synthesis and antimicrobial potential of nitrofuran-triazole congeners," Organic \& Biomolecular Chemistry 13(36), 9388-9397. DOI: $10.1039 / \mathrm{c} 5 \mathrm{ob} 01353 \mathrm{~d}$

Kukowski, K. (2018). "Role of triazoles in wood protection: Developing analytical approaches and examining sorption, distribution, and environmental fate of fungicides," Ph.D. Dissertation, University of North Dakota, Grand Forks, ND.

Kumar, D., Beena, Khare, G., Kidwai, S., Tyagi, A. K., Singh, R., and Rawat, D. S. (2014). "Synthesis of novel 1,2,3-triazole derivatives of isoniazid and their in vitro and in vivo antimycobacterial activity evaluation," European Journal of Medicinal Chemistry 81, 301-313. DOI: 10.1016/j.ejmech.2014.05.005

Liang, Z., Xu, H., Tian, Y., Guo, M., Su, X., and Guo, C. (2016). "Design, synthesis and antifungal activity of novel benzofuran-triazole hybrids," Molecules 21(6), 732-743. DOI: $10.3390 /$ molecules21060732

Lima-Neto, R. G., Cavalcante, N. N. M., Srivastava, R. M., MendonçaJr., F. J. B., Wanderley, A. G., Neves, R. P., and dos Anjos, J. V. (2012). "Synthesis of 1,2,3triazole derivatives and in vitro antifungal evaluation on Candida strains," Molecules 17(5), 5882-5892. DOI: 10.3390/molecules17055882

Mohammadi-Khanaposhtani, M., Saeedi, M., Zafarghandi, N. S., Mahdavi, M., Sabourian, R., Razkenari, E. K., Alinezhad, H., Khanavi, M., Foroumadi, A., Shafiee, A., et al. (2015). "Potent acetylcholinesterase inhibitors: Design, synthesis, biological evaluation, and docking study of acridone linked to 1,2,3-triazole derivatives,"

European Journal of Medicinal Chemistry 92, 799-806. DOI: 10.1016/j.ejmech.2015.01.044

Nazari, L., and Hosseinihashemi, S. K. (2017a). "Laboratory study of synergistic effects of mixtures of organic biocides, metal chelator, and antioxidant materials against rainbow white-rot fungus," Iranian Journal of Wood Paper and Science Research 32(3), 423-435. DOI: 10.22092/ijwpr.2017.114464.1424

Nazari, L., and Hosseinihashemi, S. K. (2017b). "Antifungal synergistic effects of organic pesticides and extracts from the inner bark of barberry (Berberis vulgaris) stem on the white-rot fungus," Journal of Renewable Natural Resource Research 7(4), 29-43.

Obanda, D. N. (2008). Biotransformation of Organic Wood Preservatives by Microorganisms, Ph.D. Dissertation, Louisiana State University, Baton Rouge, LA.

Obanda, D. N., and Shupe, T. F. (2009). "Biotransformation of tebuconazole by microorganisms: Evidence of a common mechanism," Wood and Fiber Science 41(2), 157-167.

Obanda, D. N., Shupe, T. F., and Catallo, W. J. (2008). "Resistance of Trichoderma harzianum to the biocide tebuconazole - Proposed biodegradation pathways," Holzforschung 62(5), 613-619. DOI: 10.1515/HF.2008.098

Potter, T. L., Strickland, T. C., Joo, H., and Culbreath, A. K. (2005). "Accelerated soil dissipation of tebuconazole following multiple applications to peanut," Journal of Environmental Quality 34(4), 1205-1213. DOI: 10.2134/jeq2004.0473

Reinprecht, L. (2010). "Fungicides for wood protection - World viewpoint and evaluation/testing in Slovakia," in: Fungicides, O. Carisse (ed.), InTech, London, UK, 
pp. 95-122. DOI: $10.5772 / 13233$

Shi, Y.-M., Yang, L.-J., Chen, W., Sun, C.-J., Xu, X.-L., Zhou, S.-Y., Zhang, H.-B., and Yang, X.-D. (2014). "Synthesis and biological activity of novel 1-((benzofuran-2yl)methyl)-1Htriazole derivatives," Letters in Drug Design \& Discovery 11(8), 975984. DOI: 10.2174/1570180811666140423214501

Weide, T., Saldanha, S. A., Minond, D., Spicer, T. P., Fotsing, J. R., Spaargaren, M., Frère, J.-M., Bebrone, C., Sharpless, K. B., Hodder, P. S., et al. (2010). "NH-1,2,3triazole inhibitors of the VIM-2 metallo- $\beta$-lactamase," ACS Medical Chemistry Letters 1(4), 150-154. DOI: $10.1021 / \mathrm{ml} 900022 \mathrm{q}$

White, P. M., Potter, T. L., and Culbreath, A. K. (2010). "Fungicide dissipation and impact on metolachlor aerobic soil degradation and soil microbial dynamics," Science of the Total Environment 408(6), 1393-1402. DOI: 10.1016/j.scitotenv.2009.11.012

Woo, C., Daniels, B., Stirling, R., and Morris, P. (2010). "Tebuconazole and propiconazole tolerance and possible degradation by basidiomycetes: A wood-based bioassay," International Biodeterioration \& Biodegradation 64(5), 403-408. DOI: 10.1016/j.ibiod.2010.01.009

Wüstenhöfer, B., Wegen, H. W., and Metzner, W. (1993). "Triazoles, a new fungicide generation for wood preservation," Holz-Zentralblatt 119, 984-988.

Xu, S., Zhuang, X., Pan, X., Zhang, Z., Duan, L., Liu, Y., Zhang, L., Ren, X., and Ding, K. (2013). "1-Phenyl-4-benzoyl-1H-1,2,3-triazoles as orally bioavailable transcriptional function suppressors of estrogen-related receptor $\alpha$," Journal of Medicinal Chemistry 56(11), 4631-4640. DOI: 10.1021/jm4003928

Article submitted: June 15, 2020; Peer review completed: August 23, 2020; Revised version received and accepted: August 24, 2020; Published: August 28, 2020.

DOI: 10.15376/biores.15.4.7828-7843

ERRATUM: September 1, 2020: Page 7833, $3^{\text {rd }}$ paragraph, three plates were changed to one plate. 